\title{
Self-made thoracic needled suspending device with a snare: An excellent aid for uniportal video-assisted thoracic lobectomy and segmentectomy for lung cancer
}

\author{
SHANLEI WANG ${ }^{1,2}$, CHUIZHENG MENG $^{2}$, ZHONGMIN JIANG $^{1}$, DIEGO GONZALEZ-RIVAS ${ }^{3}$, \\ JUNFEN RUAN ${ }^{2}$, WEI XU ${ }^{2}$, CHUANPING LIU ${ }^{2}$, LEI ZHANG ${ }^{2}$, \\ GUOGANG GAO ${ }^{2}, \mathrm{GE} \mathrm{YU}^{2}$, HEZHI TENG ${ }^{2}$ and JIN JU ${ }^{2}$ \\ ${ }^{1}$ Department of Thoracic Surgery, Shandong Provincial Qianfoshan Hospital, Shandong University, Jinan, Shandong 250000; \\ ${ }^{2}$ Department of Thoracic Surgery, Weihai Municipal Hospital, Weihai, Shandong 264200, P.R. China; \\ ${ }^{3}$ Department of Thoracic Surgery (Coruña University Hospital) and Minimally Invasive \\ Thoracic Surgery Unit (UCTMI), 15008 Coruña, Spain
}

Received August 28, 2018; Accepted January 15, 2019

DOI: $10.3892 / \mathrm{ol} .2019 .10030$

\begin{abstract}
Safety and feasibility of the self-made thoracic needled suspending device with a snare in the uniportal video-assisted thoracic lobectomy and segmentectomy for the treatment of non-small cell lung cancer were explored. In total, 80 pulmonary lung major resections (including lobectomy and segmental resections) with systematic mediastinal lymphadenectomy were retrospectively analyzed. Patients were randomly divided into an observation group and a control group. In the observation group, the device was used to hang affected lungs, left and right vagus nerve at the level of tracheal bifurcation, the arch of azygos vein, left phrenic nerve and left and right bronchus on the chest wall to offer a better exposure of the operation field. In the control group, the conventional uniportal video-assisted thoracic surgery was performed without using the self-made device. Systematic mediastinal lymphadenectomy was performed in both groups. Operation time, intraoperative blood loss, postoperative extubation time, hospital stay and perioperative complications in the early stage of patients in both groups were compared. The operation time
\end{abstract}

Correspondence to: Dr Shanlei Wang, Department of Thoracic Surgery, Weihai Municipal Hospital, 70 Heping Road, Weihai, Shandong 264200, P.R. China

E-mail: w2e3x4@163.com; wangshanlei@hotmail.com

Dr Zhongmin Jiang, Department of Thoracic Surgery, Shandong Provincial Qianfoshan Hospital, Shandong University, 16766 Jingshi Road, Jinan, Shandong 250000, P.R China

E-mail: qyjzm@sina.com

Key words: the self-made thoracic needled suspending device with a snare, uniportal video-assisted thoracic surgery, pulmonary lobectomy or segmentectomy, systematic mediastinal lymphadenectomy, lung cancer
$120.2 \pm 40.32 \mathrm{~min}$, intraoperative blood loss $100.51 \pm 50.23 \mathrm{ml}$, and postoperative suction drainage volume $208 \pm 97.56 \mathrm{ml} /$ day in the observation group were significantly different from those in the control group $(\mathrm{P}<0.05)$, and there were no significant differences in postoperative extubation time, hospital stay and perioperative complications between the two groups ( $P>0.05)$. The self-made thoracic needled suspending device with a snare is an excellent helper for uniportal video-assisted thoracic surgery, because it helps to expose surgical field and has no postoperative cicatrisation at puncture point on the wall of the chest. The device and its use are worthy of promotion.

\section{Introduction}

Uniportal video-assisted thoracic surgery has become one of the most important development directions of minimally invasive thoracic surgery since the first case of uniportal video-assisted thoracic lobectomy was performed in 2011 by Gonzalez et al (1). In recent years, an increasing number of thoracic surgeons have tried pulmonary lobectomy or segmentectomy in uniportal video-assisted thoracic surgery, thoracic experts have attempted sleeve lobectomy, tracheal and carinal resection and other difficult surgery.

In 2003, Migliore (2) first reported the application of uniportal video-assisted thoracic surgery in the diagnosis and treatment of non-complex pleural diseases. Rocco (3) reported uniportal video-assisted thoracic surgical resections in 2004. Miyajima et al (4) and Okada (5) reported uniportal video-assisted thoracic lobectomy and segmentectomy in 2011 and 2012, respectively. Uniportal video-assisted thoracic surgery is a trend of endoscopic surgery. Compared with the conventional thoracoscopic surgery, the incision is slightly forward, where the intercostal space is relatively wide. The impact of the operation on the intercostal nerve will be minimized, and the postoperative pain of the patient will be alleviated with minor trauma (6). Consequently, it is more acceptable to patients. Moreover, due to the smaller incision, the limited viewing field and operating space require surgeons 
with excellent skills. Different from conventional thoracoscopic surgery in the operation ideas, it requires a long learning curve to change from three-portal video-assisted thoracoscopic surgery to uniportal video-assisted thoracic surgery. As a result, only a handful of top specialists from major hospitals are performing the procedure. However, due to only 2 surgeons in uniportal video-assisted thoracic surgery, only one incision and lack of assistant, it enhances the difficulty of surgical exposure, so that it becomes a major problem of beginners to expose the surgical field in uniportal video-assisted thoracic surgery. How to get a surgical field without increasing extra distinct wound is the aim of this study. We adopted the self-made thoracic needled suspending device with a snare to help expose a surgical field in uniportal video-assisted thoracic surgery and obtained an excellent results.

\section{Patients and methods}

Patients. Eighty patients who received uniportal video-assisted thoracic surgery in Mount Qianfuo Attatched Hospital of Shandong University (Jinan, China) between July 2016 and July 2018 were enrolled. Among them, there were 52 males and 28 females, aged from 28 to 76 years. Two patients had mild cough, and other patients had no obvious clinical symptoms such as cough, fever, bloody sputum and chest pain. These patients were randomly divided into observation group and control group. Forty patients in the observation group received the operation using the self-made thoracic needled suspending device with a snare which hang affected lungs, left and right vagus nerve at the level of tracheal bifurcation, the arch of azygos vein, left phrenic nerve and left and right bronchus on the chest wall to expose surgical field in order to perform fluent operation. Another 40 patients in the control group received the conventional uniportal video-assisted thoracic surgery without using the self-made device. Systematic mediastinal lymphadenectomy was performed in both groups. Operation time, intraoperative blood loss, postoperative extubation time, hospital stay and perioperative complications in the early stage of patients in both groups were compared.

Retrospectively 80 cases of pulmonary lobectomy or segmentectomy were analyzed with systematic mediastinal lymphadenectomy in uniportal video-assisted thoracic surgery for lung cancer. In the obervation group, the device was used to hang affected lungs, left and right vagus nerve at the level of tracheal bifurcation, the arch of azygos vein, left phrenic nerve and left and right bronchus on the chest wall to offer a better exposure of the operation field. In the control group, the self-made device was not used. The assistant helped to expose the surgical field or the surgeon used an elbow aspirator to expose the surgical field. The rest of the surgical operations were the same in both groups.

This study was approved by the Ethics Committee of Mount Qianfuo Attatched Hospital of Shandong University. Signed informed consents were obtained from the patients and/or guardians.

Preparation of the self-made thoracic needled suspending device with a snare

Materials. Puncture needle (a transfusion puncture hollowed needle), snare thread (a strip of 3-0 prolene), overhanging thread (a strip of 7\# silk thread), and 8\# folded silicon ureter. The materials were easily obtained in the operation room. The self-made thoracic needled suspending device with a snare was made before surgery. Firstly using a strip of 3-0 prolene passed the transfusion puncture hollowed needle, and ligated the two ends of the 3-0 prolene, so that a prolene loop was gained. Secondly, using the transfusion puncture hollowed needle the suited point of the chest wall was prodded, then the whole needle head was made in the patient thoracic cavity. The needle was withdrawn about $1-2 \mathrm{~cm}$, so the prolene formed a snare, like a ring, at the pinhead of the hollowed needle in thoracic cavity (Fig. 1). Lastly the prolene ring was passed using a strip of $7 \#$ silk thread which pulled tissues needed, the hollowed needle was dropped, and the prolene pulled out of the chest wall. At the same time, the $7 \#$ silk thread was fixed and pulled out of the chest wall. When a long time was needed at some part in the surgery, this device was used to obtain favorable surgical field by overhanging tissue to a suitable point of the chest wall. The tissues which were often overhung were left and right vagus nerve at the level of tracheal bifurcation, the arch of azygos vein, left and right bronchus, left phrenic nerve, and diseased lungs.

Anesthesia and operative position. All patients were under general anesthesia, with double-lumen intubition and single-lung ventilation, and in full lateral decubitus position with flexion of the rotatable operation table at the level of the mid-chest, allowing slight widening of the intercostal space that improved exposure and facilitated access to the pleural cavity. In the surgery, the rotatable operation table can be rotated depending on operative need, so that satisfactory operative field can be obtained because the lung can be kept away from the surgical field due to the gravity of the lung.

Surgical technique. Using uniportal video-assisted right upper lobectomy through posterior approach with systematic mediastinal lymphadenectomy as an example, we explain how the self-made needled suspending device with snare play is used in exposing the surgical field in uniportal video-assisted surgery.

i) Making incision: A 3-5 cm incision was made in the fifth intercostal space posterior to the anterior axillary line and a wound protector was commonly placed. One end of a strip of 8 \# folded silicon ureter on the posterior side of the incision $5 \mathrm{~cm}$ away from the wound protector was sutured and fixed, and the other end was fixed on a camera cannula to facilitate the camera in and out of the chest cavity. The operation was performed 2-4 $\mathrm{cm}$ ahead. When rotated in different direction, the thoracoscope was kept on the back edge of the incision in camera cannula by the traction of elasticity of the $8 \#$ folded silicon ureter from beginning to end (Fig. 2). Consequently, there is a supporting point for camera holder, and the assistant could use one hand to handle the camera and the other hand to help exposure or sucking.

ii) Round oval forceps were used to lift part of the lung tissue, with pointed curved forceps clamp. 7\# silk thread was used to ligate part of the lung tissue, and the hollow puncture needle of the prefabricated needle snare device was used to puncture through the chest wall, so that the whole hollow needle head enters the chest cavity, and then the needle was withdrawn about 1-2 cm, the slip line forms a circle at the tip of the needle in the chest cavity. Finally, the $7 \#$ thread of the 

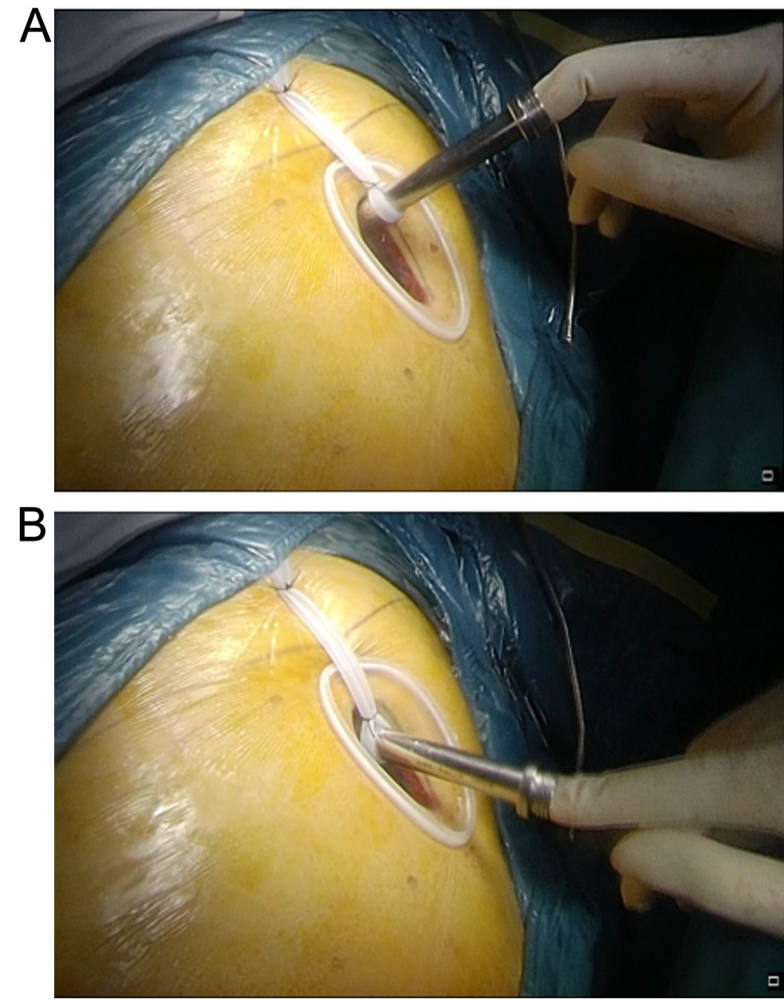

Figure 1. The hollow needle of the prefabricated device was prodded in the third intercostal chest wall at the right anterior axillary line. The needle was withdrawn about $1-2 \mathrm{~cm}$, so the prolene formed a snare, like a ring, at the pinhead of the hollow needle in the thoracic cavity. (A) The suture was fixed at one end of an $8 \#$ folded silicon ureter on the posterior side of the incision $3-5 \mathrm{~cm}$ away from the wound protector, the other end of it was fixed on the camera cannula. (B) When rotated in different directions, the thoracoscope was kept on the back edge of the incision in the camera cannula by the traction of elasticity of the $8 \#$ folded silicon ureter from beginning to end.

suspension tissue was inserted into the sliding coil in the chest cavity, and the hollow needle was removed. The slip line was pulled out of the chest wall, and the $7 \#$ thread was pulled out of the body and suspended, and the tattoo clamp was fixed. As a result, the affected lung is suspended in the appropriate direction of the chest wall, thereby exposing the operating field opposite to the suspension direction. It expanded the field of surgery. Then the conventional uniportal video-assisted thoracic lobectomy and segmentectomy were performed. Due to increased suspension-assisted exposure during the operation, the assistance through a single hole is reduced, thus reducing the interference of the instruments during the operation.

iii) By using the method of ligation or suture belt mentioned above, nerve, blood vessel, bronchus were suspended in the appropriate position of the chest wall to help the exposure of surgical field. The right vagus nerve and pleura under the carina were suspended in the posterior chest wall to expose the supraspinatus surgery field, so as to facilitate the dissection of 7 groups of lymph nodes (Fig. 3). The azygos arch was suspended in the forward, upper and lower directions to facilitate the exposure of the surgical field for the dissection of lymph nodes in groups 2 and 4 on the right (Fig. 4). The left phrenic nerve was suspended in 2 directions forward and back to facilitate the exposure of the surgical field, facilitating the dissection of the left 5 and 6 groups of lymph nodes. The

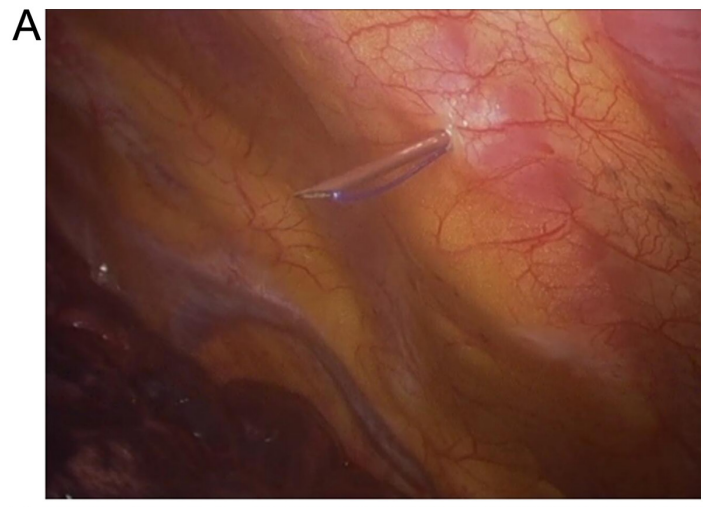

B

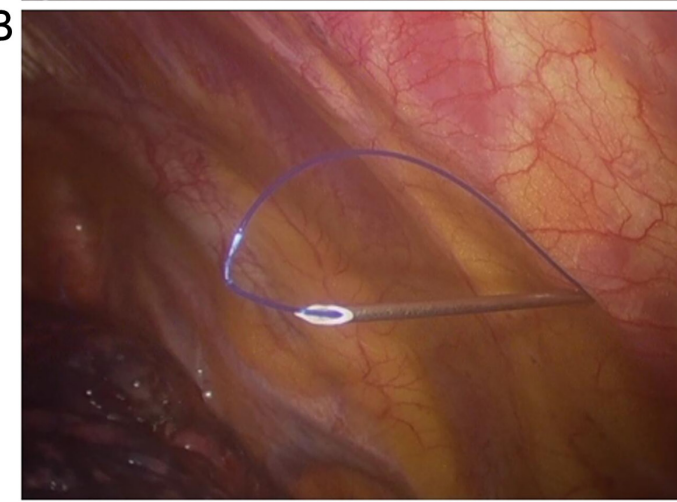

Figure 2. One end of an $8 \#$ folded silicon ureter was sutured and fixed on the posterior side of the incision $3-5 \mathrm{~cm}$ away from the wound protector, the other end was fixed on the camera cannula. When rotated in different directions, the thoracoscope was kept on the back edge of the incision in the camera cannula by the traction of elasticity of the $8 \#$ folded silicon ureter from beginning to end. (A) Using the hollow needle of the device the chest wall was prodded in the third intercostal at the right anterior axillary line. (B) The needle was withdrawn about $1-2 \mathrm{~cm}$, so the prolene formed a snare, like a ring, at the pinhead of the hollow needle in the thoracic cavity.

left main bronchus was suspended forward and exposed to the left subcarina suture field to facilitate dissection of the left 7 groups of lymph nodes. The left main bronchus was suspended forward to facilitate dissection of the left 4 groups of lymph nodes. It is suspended at the appropriate position of the chest wall to assist the exposure of the surgical field.

iv) At the end of the procedure, a strip of 20\# silicon tube was tunneled to thoracic apex through the subcutaneous, serratus anterior and the upper border of the upper lib of the incision. In addition, a central venous catheter was used to puncture chest wall into the posterior costo-phrenic angle for drainage.

Statistical analysis. SPSS 16.0 (SPSS, Inc., Chicago, IL, USA) statistical software was used to analyze the data. The measurement data were represented as mean $\pm \mathrm{SD}$, and t-test was used for comparison. The comparison of count data was performed using $\chi^{2}$ test. Difference at $\mathrm{P}<0.05$ was considered to be statistically significant.

\section{Results}

Of the 80 patients, 52 were males and 28 females, 50 patients underwent pulmonary lobectomy, and 30 patients underwent pulmonary segmentectomy. There were 76 patients with adenocarcinoma, 3 with squamous cell carcinoma, and 1 with 
Table I. Comparison of uniportal video-assisted thoracic lobectomy and segmentectomy for lung cancer between two groups.

\begin{tabular}{|c|c|c|c|}
\hline Items & $\begin{array}{l}\text { Control } \\
\text { (without the self-made } \\
\text { thoracic needled suspending } \\
\text { device with a snare) }\end{array}$ & $\begin{array}{l}\text { Observation } \\
\text { (with the self-made } \\
\text { thoracic needled suspending } \\
\text { device with a snare) }\end{array}$ & P-value \\
\hline Case (n) & 40 & 40 & - \\
\hline Operation time (min) & $140.5 \pm 60.45$ & $120.2 \pm 40.32^{\mathrm{a}}$ & 0.006 \\
\hline Intraoperative blood loss (ml) & $130.87 \pm 52.63$ & $100.51 \pm 50.23^{\mathrm{a}}$ & 0.001 \\
\hline Postoperative suction drainage volume (ml/days) & $230.62 \pm 100.57$ & $208 \pm 97.56^{\mathrm{a}}$ & 0.008 \\
\hline Postoperative extubation time (days) & $2.8 \pm 2.14$ & $2.1 \pm 1.24^{\mathrm{a}}$ & 0.005 \\
\hline Hospital stay after operation (days) & $7.1 \pm 3.21$ & $7.3 \pm 2.17$ & 0.07 \\
\hline Convert to three port video-assisted thoracic surgery & 4 & 2 & 0.09 \\
\hline Convert to thoracotomy (n) & 0 & 1 & - \\
\hline Serious complications & 0 & 0 & - \\
\hline
\end{tabular}

${ }^{\mathrm{a} C}$ Compared with the control group, $\mathrm{P}<0.05$.

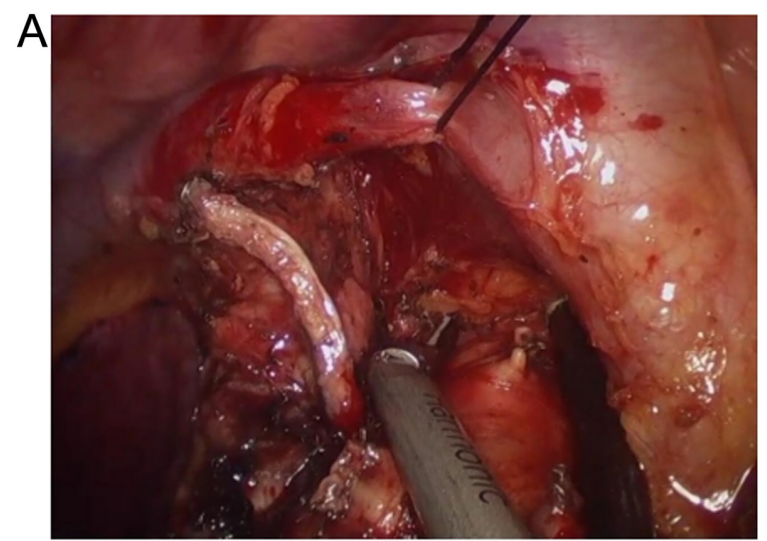

$\mathrm{B}$

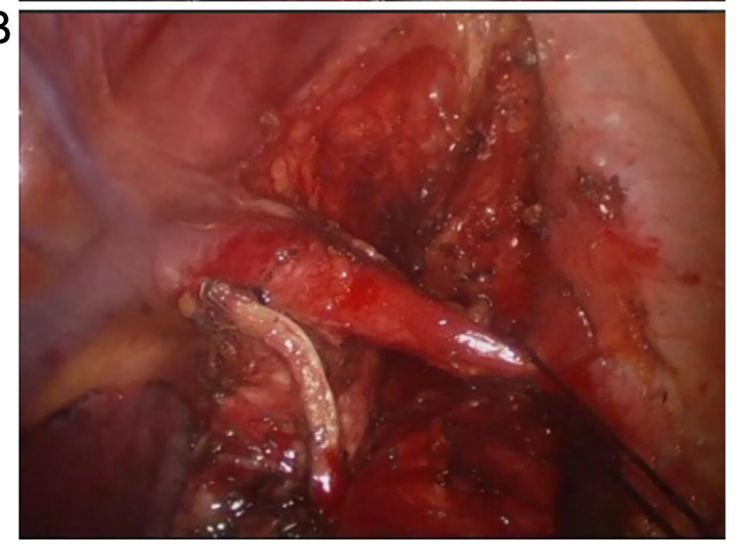

Figure 3. Suture of the posterior mediastinal pleura and the vagus nerve using a round needle with a strip of $7 \#$ silk thread pulled out of the chest wall in the sixth intercostal at the scapular line by the self-made needled suspending device. The area under trachea carina was enlarged when the $7 \#$ silk thread was tightly pulled and the lower right lobe was pushed forward by sponge forceps. (A) The arch of azygos vein was overhung forward and upward by the device. (B) The arch of azygos vein was overhung forward and downward by the self-made needled suspending device.

small cell carcinoma. Two patients converted to three port video-assisted thoracic surgery because of extensive adhesion. One patient converted to thoracotomy on account of middle lobar venous hemorrhage, and the conversion rate was $2.5 \%$.

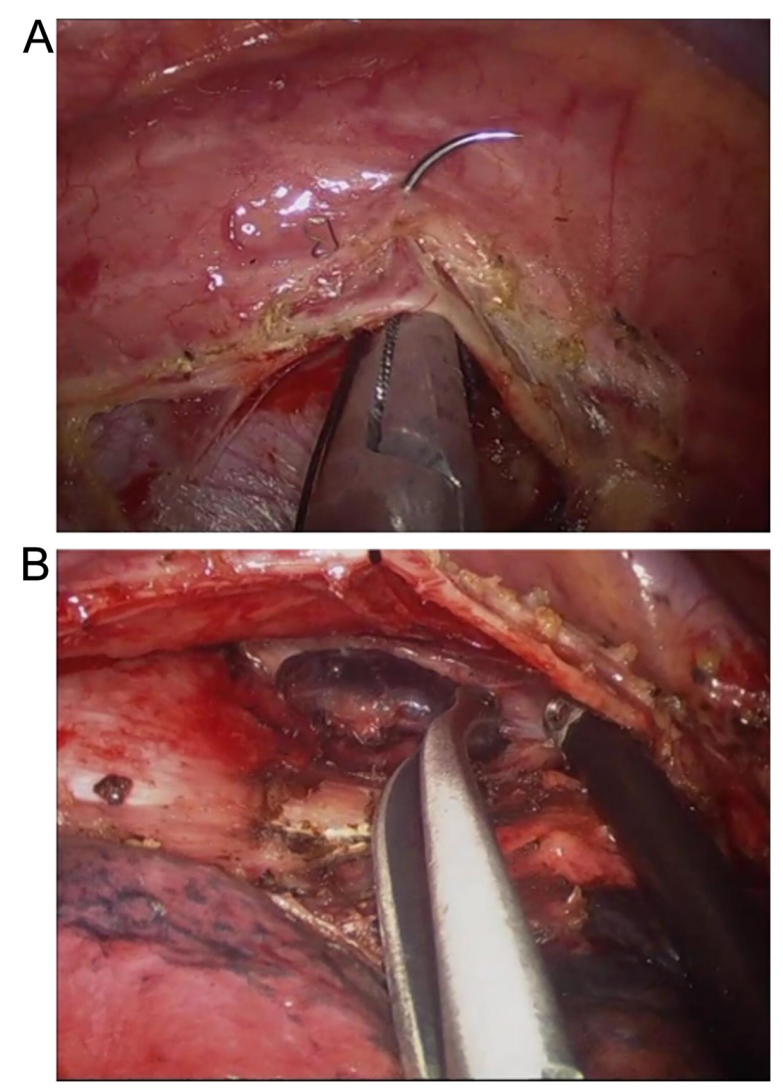

Figure 4. The arch of azygos vein was overhung forward and upward by the self-made needled suspending device. (A) Suture of the posterior mediastinal pleura and the vagus nerve using a round needle with a strip of 7\# silk thread pulled out of the chest wall in the sixth intercostal at the scapular line by the self-made needled suspending device. (B) The area under trachea carina was enlarged when the $7 \#$ silk thread was tightly pulled and the lower right lobe was pushed forward by sponge forceps.

The rest received successful uniportal video-assisted thoracic lobectomy and segmentectomy as well as systematic mediastinal lymphadenectomy, without serious complications or surgical death. The operation time $120.2 \pm 40.32 \mathrm{~min}$, 
intraoperative blood loss $100.51 \pm 50.23 \mathrm{ml}$, and postoperative suction drainage volume $208 \pm 97.56 \mathrm{ml} /$ day in the observation group were significantly different from those in the control group $(\mathrm{P}<0.05)$, and there were no significant differences in postoperative extubation time, hospital stay and perioperative complications between the two groups $(\mathrm{P}>0.05)$ (Table I).

\section{Discussion}

Compared with three portal video-assisted thoracic surgery, the vice operation hole of posterior axillary line and the observation hole of axillary midline are not needed in the uniportal video-assisted thoracic surgery. The incision was between 4 th and 5 th ribs of anterior axillary line with wide rib clearance, few chest wall muscle layers and high elasticity, so the bleeding is easy to be stopped, which leads to smaller damage to the body, less postoperative pain and less impact on the patients' sensation and movement (7). Chen et al (8) compared the clinical effects of 20 cases of three portal video-assisted thoracic surgery and 10 cases of uniportal video-assisted thoracic surgery in the treatment of pulmonary interstitial diseases, and found that the VAS score of pain in the uniportal video-assisted thoracic surgery group on the first day after surgery was $4.5 \pm 0.7$ points significantly lower than that of the three portal video-assisted thoracic surgery group $4.95 \pm 0.39$ points with a statistically significant difference $(\mathrm{P}=0.03$. Bertolaccini et al (9) considered that unlike the three portal video-assisted thoracic surgery, the uniportal video-assisted thoracic surgery was closer to traditional open surgery. Gonzalez-Rivas (10) reviewed the evolution of thoracic surgery from open surgery, assisted thoracoscopic surgery, total thoracoscopic surgery, the uniporta thoracic surgery to the uniportal video-assisted thoracic surgery, and believed that the perspectives of uniportal video-assisted thoracic surgery and open surgery were basically similar.

Although it has more advantages in contrast with three port surgery, it has its own disadvantages - the problem of surgical exposure and the problem of instrumental mutual interference. A retrospective analysis was performed on 40 patients who underwent pulmonary lobectomy or segmentectomy with systematic mediastinal lymphadenectomy in uniportal video-assisted thoracic surgery for lung cancer. Self-made thoracic needled suspending device with a snare was detailed in making and the method with respect to this device, and right upper lobectomy and right mediastinal lymphadenectomy in uniportal video-assisted thoracic surgery were exampled for specific use of this device. Compared with the conventional uniportal video-assisted thoracic surgery, it has distinct advantages on blood loss and operation time.

Uniportal video-assisted thoracic surgery was performed with the self-made thoracic needled suspending device with a snare and was referred to as suspending uniportal video-assisted thoracic surgery in this report. During the operation, the self-made thoracic needled suspending device with a snare was used to hang affected lungs, left and right vagus nerve at the level of tracheal bifurcation, the arch of azygos vein, left phrenic nerve and left and right bronchus on the chest wall in uniportal video-assisted thoracic surgery to expose surgical field in order to perform fluent operation. It was like a hand added in the conventional uniportal video-assisted thoracic surgery to facilitate the operation. The hand could help in different directions in different locations on the chest wall to help the fluent operation in various ways. This hand is invisible, and does not pass through the chest wall incision. It only leaves a pinprick on the chest wall which will not leave a scar after surgery. This method can effectively offset the disadvantages of the uniportal video-assisted thoracic surgery, including the mutual interference of surgical instruments and the poor vision of the conventional surgery. Since ligation and suture belt are used to fix target tissue, it takes a certain time. To reduce the time of operation, pulmonary forceps of the thoracoscope with a line will be used in the future. Robotic surgery is a development direction of the minimally invasive thoracic surgery and this method can be regarded as low cost uniportal video-assisted thoracic robotic surgery, because the mechanical hand can work in multiple directions. Like the uniportal video-assisted thoracic robotic surgery, this method could be an option for the thoracic surgery. Since this study focuses on the feasibility of the surgical method, it has some limitations. Long-term efficacy was not examined. Besides, this study is a single center study, and multi-center study is still required.

In conclusion, the self-made thoracic needled suspending device with a snare is an excellent helper for uniportal video-assisted thoracic surgery, because it helps to expose surgical field and has no postoperative cicatrisation at puncture point on the wall of the chest. The device and its use are worthy of promotion.

\section{Acknowledgements}

Dr Shanlei Wang is grateful to Professor Diego Gonzalez-Rivas for uniportal video-assisted thoracic surgery training and wish to express his gratitude to Professor Song Zhao, Professor Ge-Ning Jiang and Professor Zhong-Min Jiang for mentoring.

\section{Funding}

No funding was received.

\section{Availability of data and materials}

The datasets used and/or analyzed during the current study are available from the corresponding author on reasonable request.

\section{Authors' contributions}

SW, ZJ and DGR conceived the study and drafted the manuscript. JR, WX, CM and CL acquired the data. LZ, GG and GY analyzed the data. HT and JJ revised the manuscript and were responsible for statistical analysis. All authors read and approved the final manuscript.

\section{Ethics approval and consent to participate}

This study was approved by the Ethics Committee of Mount Qianfuo Attatched Hospital of Shandong University (Jinan, China). Patients who participated in this research had complete clinical data. The signed informed consents were obtained from the patients or the guardians. 


\section{Patient consent for publication}

Not applicable.

\section{Competing interests}

The authors declare that they have no competing interests.

\section{References}

1. Gonzalez D, Paradela M, Garcia J and Dela Torre M: Single-port video-assisted thoracoscopic lobectomy. Interact Cardiovasc Thorac Surg 12: 514-515, 2011.

2. Migliore M: Efficacy and safety of single-trocar technique for minimally invasive surgery of the chest in the treatment of noncomplex pleural disease. J Thorac Cardiovasc Surg 126: 1618-1623, 2003.

3. Rocco G: One-port (uniportal) video-assisted thoracic surgical resections - a clear advance. J Thorac Cardiovasc Surg 144: S27-S31, 2012

4. Miyajima M, Watanabe A, Uehara M, Obama T, Nakazawa J, Nakajiima T, Ogura K and Higami T: Total thoracoscopic lung segmentectomy of anterior basal segment of the right lower lobe (RS8) for NSCLC stage IA (case report). J Cardiothorac Surg 6: 115, 2011.
5. Okada M: Identification of intersegmental border in radical segmentectomy. Kyobu Geka 63 (Suppl): 697-701, 2010 (In Japanese)

6. Ren M, Meng Q, Zhou W, Kong F, Yang B, Yuan J, Wu D, Zhang J, Li Q, Lin Y, et al: Comparison of short-term effect of thoracoscopic segmentectomy and thoracoscopic lobectomy for the solitary pulmonary nodule and early-stage lung cancer. Onco Targets Ther 7: 1343-1347, 2014.

7. Shapiro M, Weiser TS, Wisnivesky JP, Chin C, Arustamyan M and Swanson SJ: Thoracoscopic segmentectomy compares favorably with thoracoscopic lobectomy for patients with small stage I lung cancer. J Thorac Cardiovasc Surg 137: 1388-1393, 2009.

8. Chen PR, Chen CK, Lin YS, Huang HC, Tsai JS, Chen CY and Fang HY: Single-incision thoracoscopic surgery for primary spontaneous pneumothorax. J Cardiothorac Surg 6: 58-58, 2011.

9. Bertolaccini L, Rocco G, Viti A and Terzi A: Geometrical characteristics of uniportal VATS. J Thorac Dis 5 (Suppl 3): S214-S216, 2013.

10. Gonzalez-Rivas D: Evolving thoracic surgery: From open surgery to single port thoracoscopic surgery and future robotic. Chin J Cancer Res 25: 4-6, 2013.

This work is licensed under a Creative Commons Attribution-NonCommercial-NoDerivatives 4.0 International (CC BY-NC-ND 4.0) License. 